\title{
Online epistemic communities: theoretical and methodological directions for understanding knowledge co-elaboration in new digital spaces
}

\author{
Françoise Détienne $^{\mathrm{a}^{*}}$, Flore Barcellini ${ }^{\mathrm{b}}$, Michael Baker $^{\mathrm{a}}$, Jean-Marie Burkhardt ${ }^{\mathrm{c}}$, Dominique Fréard ${ }^{\mathrm{a}}$ \\ ${ }^{a}$ LTCI,UMR 5141 CNRS - Telecom ParisTech, 46 rue Barrault, 75013 Paris, France \\ ${ }^{b}$ CRTD, CNAM, 41 rue Gay Lussac, 75005 Paris, France \\ ${ }^{c}$ LATI, University Paris Descartes, 71, avenue Edouard Vaillant, 92774 Boulogne Billancourt, France
}

\begin{abstract}
.
This paper presents, illustrates and discusses a generic framework for studying knowledge co-elaboration in online epistemic communities ("OECs"). Our approach is characterised by: considering knowledge co-elaboration as a design activity; distinguishing discussion and production spaces in OECs; characterising participation via the notion of role; fine-grained analyses of meaning, content and communicative functions in interactions. On this basis, three key issues for ergonomics research on OECs are discussed and illustrated by results from our previous studies on OSS and Wikipedia. One issue concerns the interrelation between design (task) and regulation. Whereas design task-oriented activity is distributed among participants, we illustrate that OCEs function with specialised emerging roles of group regulation. However, the task-oriented activity also functions at an interpersonal level, as an interplay of knowledge-based discussion with negotiation of competencies. Another issue concerns the foci of activity on the (designed) knowledge object. Based on a generic task model, we illustrate asymmetry and distinctiveness in tasks' foci of participants. The last issue concerns how design-use mediation is ensured by specific forms of mediation roles in OECs. Finally we discuss the degree of generality of our framework and draw some perspectives for extending our framework to other OECs.
\end{abstract}

Keywords: online communities, distributed design, interactive roles

\section{Introduction}

Online epistemic communities ("OECs") are new forms of distant computer-mediated cooperative work oriented toward the co-elaboration of some knowledge objects, such as encyclopaedia articles in Wikipedia, or software in Open Source Software ("OSS") communities. The main objective of this paper is to establish theoretical and methodological approaches for achieving a deep understanding of processes underlying these situations of knowledge co-elaboration. In this paper, we will first develop a generic framework for understanding design coelaboration in OECs. On this basis, we will develop and discuss three key issues for ergonomics research on OECs: (1) understanding the interplay between design (task) and regulation; (2) understanding the foci of activity on the (designed) knowledge object and; (3) design-use mediation. We will illustrate these issues by drawing on our previous research on two cases of OECs: the Python OSS project, and the Wikipedia Astronomy (French) project. In conclusion we will discuss the degree of generality of our framework and bring out some perspectives for extending our framework to other OECs.

\footnotetext{
* Corresponding author, E-mail: francoise.detienne@telecom-paristech.fr
} 


\section{A generic Framework for studying knowledge co-elaboration in OECs}

\subsection{Knowledge co-elaboration as a design activity}

For almost a decade, we have been developing a generic framework for the study of co-elaboration activities across these new digital spaces, on the basis of studies on OSS [2-5, 21], and Wikipedia [14]. Our position is to view knowledge co-elaboration in these OECs as a design activity distributed across several 'spaces' on the Internet. Indeed, design problems, classically considered as ill-structured $[10,24]$, are characterised by: many degrees of freedom in the problem's initial state; unstable design requirements in terms of constraints and subgoals; co-evolution between problem-solutions(s); various (and sometimes numerous) possible solutions with no definite way for choosing the best one. Furthermore, design is considered as a social process. Design solutions are not only based on purely technical problem-solving criteria. Design is a process of negotiating among disciplines [7].

In OSS and Wikipedia projects the collective activity mainly consists in the co-elaboration of knowledge objects, semiotic productions such as software or encyclopaedia articles, which can be viewed as the results from design choices and compromises among participants, either experts or novices, professionals or amateurs, from various disciplines.

\subsection{Distinction between production and discussion spaces}

In a generic way, we have distinguished between production spaces (code editing space in OSS, via sub-versionning, and editing of articles in Wikipedia) and discussion spaces (mailing lists or forum in OSS, editions in discussions in Wikipedia) $[14,21]$ as partially illustrated in Figure 1 below. Indeed, activity in OECs can be viewed as distributed into actions in order to reify the knowledge artefact in the production space, and communication, information sharing and also vivid debates, related to these epistemic objects in the discussion space. Both editing actions and communication activity are involved in the coelaboration of meaning and the evolution of the artefact or knowledge object.

\subsection{Characterisation of participation via the notion of role}

Understanding collaboration requires a finegrained qualitative analysis of actions in epistemic communities, as a basis for a quantitative analysis of roles and their relations. Based on analysis of interactions, through the use of coding schemes, the identification of roles corresponds to distinctive and regular individual behaviours emerging in the interaction.

Our specific focus on fine-grained content analysis and emerging roles is original in comparison with other studies on OECs. A significant body of research now exists on the structural characteristics of such communities, seen as social networks ("SNA", e.g. [25]) and on results of qualitative approaches based on questionnaires or interviews (e.g. [6]), as well as approaches that combine both (e.g. [17]). SNA approaches typically process large amounts of data, drawing on who replies to whom, as well as who edits what. However, such approaches, we would claim, largely neglect content, meaning and socio-relational relations between participants in communities. For example, the existence of exchange of messages between participants does not necessarily imply genuine collaboration between them, in the sense of a continued attempt to create shared meaning.

\subsection{Dialogical and epistemic dimensions of roles}

Our approach to analysing roles is based on the distinction between epistemic (types of knowledge evoked) and dialogical (communicative function) dimensions of contributions. This enables the identification of roles with respect to each dimension as well as to their combination. This approach was first adopted in our study of an architectural design meeting [1]. We developed an analytical framework that aimed to bring out the nature of participants' contributions in a way that distinguished content (epistemic) and communicative function dimensions (argumentation and "voices"). We termed the resulting global vision of contributions, combining roles across different dimensions, the interactive profile. This approach has been further developed to study roles in online OSS communities [4] and Wikipedia [14]. Finally, a simplified coding scheme has been developed to distinguish dialogic functions (informative, argumentative, regulative) and epistemic dimension (oriented to the task domain, e.g. Astronomy, Wikipedia) or the interpersonal domain (group, individuals). 


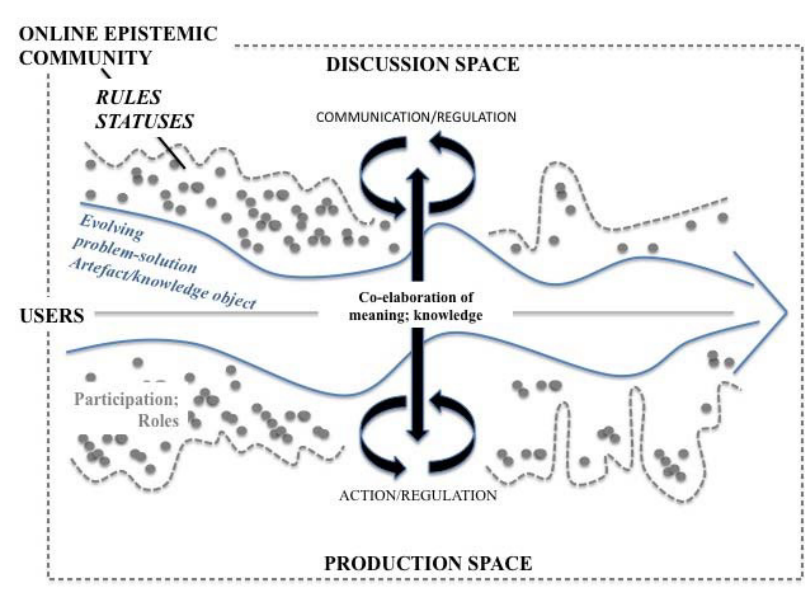

Figure 1.

Generic model for activity in epistemic online communities

\section{Online epistemic communities : Case studies on OSS-Python and Wikipedia-Astronomie}

We have carried out two case studies on an OSS project, Python, and on a Wikipedia Project, Astronomy.

Our OSS case study deals with the Python project, dedicated to the design and the use of the Python programming language. It is a dynamic and stable project spanning various application domains (scientific, game, biology, financial, web..). Within the Python community, design is framed by a specific process called the Python Enhancement Proposal (PEP), used to propose new functionalities underlying evolutions of the language. We assume that design-related data are PEP interactions distributed across a discussion space (use-oriented and design-oriented mailinglists in this case) and a production space. Indeed, once it has been proposed, a PEP is discussed in the discussion space, and the functionality proposed by the PEP is implemented in the production space. The "champion" is a special status attributed to the proposer and person who is responsible of a PEP.

We analysed a PEP process [2,3] dealing with the introduction of a new way of programming with decimals in Python, which is an important issue for various application domains. Our corpus covers the complete period of the debate (September 2003 to may 2006). Up to 95 participants discussed in 51 discus- sions (22 in the use-oriented mailing-list and 29 in the design-oriented mailing-list).

Our Wikipedia case study [14] concerns a conflict in the Astronomy project of the French-speaking Wikipedia. Astronomy is typically a domain of interest for professional scientists as well as for amateurs and the general readership, or 'everyman'1. Online discussions can thus be very vivid between participants, bringing out various epistemic domains of reference.

Our corpus was based on the discussion and article edits of the "Pluto" article. The conflict that we analysed concerned whether to follow the new academic nomenclature ("(134340) Pluto") for the title page, or to use a specific title (e.g. "Pluto (dwarf planet)"). Our corpus covers the complete period of the debate (August 2006 to June 2008). Forty participants discussed in 18 threads and produced 202 interventions in the complete debate. Two of them were researchers in Astronomy.

These two corpora were constructed and coded manually.

\section{Understanding the interrelation between design (task) and regulation}

Studies on group work emphasise the distinction between group-oriented and task-oriented activities in a collaborative framework. Based on this classical distinction, our case studies have shown how design and regulation activities are distributed and intertwined in OECs.

\subsection{Specialised emerging roles of regulation}

In our case study on the Python project, the exchanges in the discussion space were coded so as to distinguish between dialogical and epistemic dimensions of participation. This enabled us to contrast task-related and group-oriented roles in the interaction.

Task-related roles (design) are characterised by: discursive functions related to design (proposal, evaluation, clarification), and epistemic functions related to providing knowledge (application domain, computer science, scenario of use ...).

\footnotetext{
${ }^{1}$ We choose here the term "everyman" to designate the point of view of "anyone and everyone" (here, general readers of wikipedia) given, precisely, its reference to a specific theatrical role in Mediæval English morality plays (http://en.wikipedia.org/wiki/Everyman_(play)).
} 
Group-oriented role are characterised by: discursive functions related to group management or regulation (coordination, decision, synthesis), and discursive functions related to interpersonal relationships (acknowledgment of others' work, support...).

Globally, we found that the amount of designoriented activities is more important than grouporiented activities. In this respect, the distribution between task and regulation is quite similar to the one observed in previous studies of software design in collocated or distant situations (e.g. $[12,18]$ ).

We also examined whether or not there are specific roles oriented towards design or regulation. Whereas the design task-oriented activity is distributed amongst all the participants involved in the discussions, regulation (of the task and the discussion itself) is restricted to a few participants. Thus there are specialised emerging roles of regulation played by a few participants, some of whom have specific statutes (the project leader and the champion) whilst others are not characterised by specific rights or responsibilities in the project.

\subsection{Interplay of knowledge-based discussion with negotiation of competencies}

In our case study on the Wikipedia Astronomy project [14], we have highlighted that the argumentative dialogue proceeds by a subtle interplay between arguments drawing on different types of knowledge (scientific, everyday) and the images of their respective competencies that both specialists and enlightened 'amateurs' project in the interaction. Thus, the way this online epistemic community functions goes beyond knowledge-based discussion and argumentation, to involve negotiation of competencies of socalled 'experts' and 'amateurs'. This can be illustrated by an excerpt of a thread entitled "Pluto is no longer a planet" following the International Astronomical Union (IAU) decision to modify the definition of "planet" and to re-classify "planet Pluto" as an "asteroid".

One participant wrote "ITV. Pluto is no longer a planet but a star.". The reaction of another participant is: "Only a completely ignorant person would say Pluto is a star.". This reply illustrates the way participants can judge - negatively in this case the competence of other participants in the project.

\section{Understanding the foci of activity on the (designed) knowledge object}

We view collaboration fundamentally as interactions with respect to a shared task focus [20]. In OECs, participants' foci may be more or less distributed across various aspects of the knowledge object. Analysing the participants' foci of activity and their interrelations is a way to understand how distributed design proceeds. Here the interrelation between foci of activity and respective participants' statuses is the main question.

Our approach is based on a generic task model of the designed artefact itself. The structure of the artefact, and the interrelation between its parts (possibly hierarchically organised) is a layout for understanding foci of activity in the discussion spaces or the production spaces, and for addressing the issue of possible distinctive foci with respect to participants' status in the emerging organisational structure. This approach is illustrated in the subsections by our case study on Wikipedia, in which the article's structure is the layout of a generic task model.

In OSS (and software global design), a similar approach has been developed for understanding the interrelations between the artefact structure (software architecture) and the organisational structure (e.g. [16]), to provide information on coordination requirements.

\subsection{Task model in Wikipedia}

Our task-modelling approach, in progress, consists in classifying all actions in production and discussion spaces according to a common task model, distinguishing generic tasks related to the knowledge object macrostructure (structure) or microstructure (surface). These task definitions are inspired by classical frameworks on processes involved in text production (e.g. [15]) as well as in design (conceptual and detailed design).

In our case study on the Wikipedia Astronomy project, eight sub-tasks were distinguished for coding the participants' activity in the production space (editing the Pluto article) and in the discussion space (topic of posted message): (1) title edit, (2) introduction paragraph writing, (3) content writing, (4) sources citation, (5) cited pages referencing, (6) illustrations and tables edit, (7) formatting (orthography, syntax) and (8) vandalisms and their treatment. Task coding consisted in associating one of these sub-tasks to edits in article and discussion spaces. 
Whereas some subtasks are related to the article macrostructure, in particular the title and the introduction in which aim and organisation of the article is displayed, other subtasks clearly relate to the microstructure, e.g. formatting.

\subsection{Asymmetry of foci in discussion versus editing spaces and participants' distinctive task foci}

Based on this task model, our preliminary analysis of the Wikipedia Pluto article highlights an asymmetry of sub-task distribution in the article space and the discussion space. Whereas the topics of discussion concern only three subtasks - title, introduction and content - the article edits focus on all subtasks. This supports the importance of the title and introduction as framing the organisation and access to the article.

Our analysis also highlights the distinctive task foci of participants. Specialised foci were identified for three types of participants: administrators, IP addresses (non registered participants) and Robots. Administrators' foci in the discussion space mainly concerned the title and the introduction: they participated little or not at all in other subtasks. Again, the title and the introduction are judged to be of main importance, since they form the macrostructure that impacts on how the article will be accessed and first read in Wikipedia. IP addresses are responsible for most of the vandalisms. Robots were focused on editing of referencing tasks. By contrast, other discussant participants had no specific foci, being involved in all the tasks.

\section{Mediation between knowledge co-elaboration and « use »}

Another way to understand collaboration is to analyse how various sources of knowledge are mobilised and expressed by participants in OEC's collective design activity. Our approach relies on distinguishing different epistemic types of knowledge expressed in the content of the (dialogical) interactions. This is a view that is orthogonal to the former one, as discussed above, even though these epistemic distinctions could be related, on some occasions, to the structure of the artefact (as it will be the case in our example in Wikipedia).

Our approach is illustrated by the way knowledge about respectively design and use domains is mobilised and how mediation between these domains is ensured. Indeed, the quality of knowledge production in online epistemic communities is strongly related to how knowledge on "users" (users of the software or readers of Wikipedia) and cases of usage are taken into account in the knowledge co-elaboration process.

\subsection{A fuzzy distinction between users and developers}

In our case study on the Python project, the model underlying the typology of participants is based on statuses, and refers to the classical distinction between users and developers in the epistemology of design. However, one can wonder to what extent this typology corresponds to the model of participation in OSS design. Indeed, we conducted interviews in which OSS participants consistently reported that status is not relevant as a principle for distinguishing between participants' roles [4]. Furthermore, there was no agreement between participants on the meaning of the term "developer". Some interviewees referred to developers as participants in the designoriented mailing-lists, others to technical contributors, others to participants that owned rights to modify the code, or even to "those members who contributed "significantly" to the implementation of the OSS code".

Consequently, our position is that distinguishing between participants on the basis of their epistemic productions, i.e. on their epistemic roles, would be a better way to clarify mediation between design knowledge and use knowledge than the classical designer/user distinction.

Our research aim is thus to address this issue through the analysis of (1) the epistemic dimensions of discussions: knowledge domains referred to (e.g. evocation of use scenarii or readership modelling) and, (2) the distinction between specific discussion spaces (e.g. the distinction between design-oriented and use-oriented discussion lists in OSS). On this basis, we can identify specific forms of mediation roles such as boundary spanners between use and design discussion spaces in OSS (Barcellini et al. 2008a) or distinguish "everyman spokespersons" from "wikipopularisers" in Wikipedia.

\subsection{Boundary spanners in OSS}

Concerning design-use mediation in OSS project, we pointed out the presence of boundary spanners in use-design mediation. These boundary spanners are participants who traverse both use-oriented and design-oriented spaces of discussions, e.g. two discussion lists, use and development. These transversalparticipants post messages in similar topic discus- 
sions occurring in parallel, in mailing lists directed towards use or design. They have specific positions in discussions (central, summits of cliques) and form the central hub of interactions considering these positions and their active contributions.

This profile is also characterised by distinctive epistemic contribution in the discussions. They provide and share knowledge about both the use-oriented application domain and the design-oriented programming domain. Furthermore, this is carried out in an adaptative way [2]: according to the space in which these participants are evolving they transfer knowledge about end-usage to the design-oriented discussion list, for instance, and they maintain a strong focus on usage in the design-oriented discussion list.

We found that a small number of key participants act as boundary spanners. They are not necessarily users themselves: two of them were users (whilst also being developers of Python-based applications), the three others were administrators and developers of Python.

\subsection{Everyman spokeperson and wikipopulariser in Wikipedia}

In Wikipedia, the profiles of everyman spokesperson and wikipopulariser ensure mediation between design and use. In our corpus we found that some participants tended to try to impose discussion topics on:

(1) how the user, a non-expert in astronomy, classifies Pluto. The corresponding profile is one of an "everyman spokesperson", characterised by an epistemic contribution on non-expert Astronomy.

(2) how Wikipedia users navigate to find out article entries. The corresponding profile is "wikipopulariser", characterised by an epistemic contribution on non-expert wikipedia users.

The excerpts below illustrate these profiles. It is worth noting that these contributions occur in the article title discussion, thus linking a reflective activity on use and access to a macrostructure subtask, the Title subtask.

The everyman spokesperson role is illustrated by the following excerpts from a quite vivid debate.

"Me, I can see a very clear difference and I think you can notice this difference. As the lady working in your local bakery shop what Ceres is, she'll ask you if you're talking about Xeres vinegar. Talk to her about Pluto, she'll reply that she doesn't know anything about all that stuff turning around the Earth in the sky. In fact, il seems that we don't really know what it is, we've just classified it as a new planet. There, that's a planet, that's what they've just announced on TV recently. I'm making a caricature, perhaps, but sincerely, not all that much. Over these last years, school books and the "collective consciousness": aren't these the clear differences that apply to Pluto? I think your not really aware of to what extent your "known" is limited to specialists. (I didn't know about the things you spoke of, even though I have a high level scientific training). There's not one kid who hasn't heard of the name Pluto. It's a "given" that spreads over all the civilised population of the planet Earth."

"No, it's quite coherent. The lady in the bakery who comes across an article called "(134340 Pluto" will think she made a mistake in her search. By way of comparison, a user who wants information on Titan, since he's seen images of that satellite on the TV news, will go to Titan (moon). If we had directed him towards "S VI Titan", he'd ask himself questions".

"And an unfavourable opinion, I'm against it! We use the official denomination, full stop. To recall the example of the lady in the bakery, it's enough that the TV talks about it, and she'll be aware of it, so we would change names with each wave of fashion? In addition, I'm personally against pages " $x x x$ (homonymn) the notion of eclipse of meaning by another being really too subjective".

"I'm still against! One has to be consistent, to come back to the woman in the bakery, she doesn't give a damn about Pluto (it's just a little pebble far away). This article (even though it must remain understandable for everyone) is more destined for people who know about it, notably astronomers, they use the official denomination. Up to a point, I can imagine transgressing for Pluto (dwarf planet), but absolutely not for Pluto full stop! (we've just discussed the same thing for Mars/planet Mars/Mars (planet) (the second name won through)."

The wikipopulariser role is illustrated by the following excerpts in which how users access an article via its title is questioned:

"The title of an article is not the Truth, but a means for bringing users to the article, which itself must teach them the Truth. We're not speaking here about the content of the article."

"What would be interesting would be to know what the users type in order to arrive at this page. Are the statistics available?" 


\section{Discussion and perspectives}

Our framework has proved to have explanatory power for understanding knowledge co-elaboration in online epistemic communities such as OSS and Wikipedia. In future work we will extend our experimental field of study to understanding knowledge coelaboration in a broader range of communities, where diversity of knowledge, innovation and creativity [9] are emphasised, which is definitively not the case in Wikipedia, and is at best marginal in highly operational OSS communities. Whereas the aspect of our framework concerning dialogical and epistemic functions has proved useful so far, it may need to be extended for understanding (1) creativity mechanisms [19] such as far and close analogies, idea association/restructuring, multiple perspective taking and mechanisms for constructive debates, as well as (2) practical ways for articulating and structuring idea production (creativity) and value creation (diffusion).

In Wikipedia itself, we aim to extend our work to the consideration of the co-elaboration of articles whose epistemic content is not at all stabilised, such as those involving scientific and ideological controversies (e.g. evolution of species, Darwinism vs. Creationism). Here the precise and complex nature of the referent itself should be taken into account in order to gain better understanding of the collective epistemic activity.

In addition to these future extensions of the range of online epistemic communities to be studied, a further development of this research concerns using the generic model of collective activity as the theoretical basis for the study of the quality of collaboration [8, $13,22,23]$. Quality of collaboration [11] can be understood in terms of the quality or qualities of the process of working together, in terms of its outcomes (the quality of the 'output' knowledge object/artefact) and in the relation between the two (is good quality collaboration only that which produces a high quality output product?). It can also be understood from participants', users' and researchers' points of view, subjectively, 'objectively', descriptively, and normatively. These aspects can be studied by deeper analysis of the processes of negotiation of meaning and knowledge, within and across production and discussion spaces, in relation to the evolving artefact.

\section{Acknowledgements}

This research was funded by the French National Research Agency (ANR: CCCP-Prosodie project). The examples based on the Python OSS case study are drawn from Flore Barcellini's Ph.D thesis.

\section{References}

[1] M. Baker, F. Détienne, K. Lund and A. Séjourné, Etude des profils interactifs dans la conception collective en architecture., In: Détienne, F., Traverso, V (Eds) Méthodologies d'analyse de situations coopératives de conception: le corpus MOSAIC, Nancy : PUN, 2009.

[2] F. Barcellini, F. Détienne and J-M. Burkhardt, User and developer mediation in an Open Source Software Community: boundary spanning through cross participation in online discussions, International Journal of Human Computer Studies 66(7) (2008), 558-570.

[3] F. Barcellini, F. Détienne and J-M. Burkhardt, Participation in online interaction spaces: design-use mediation in an Open Source Software community, International Journal of Industrial Ergonomics, 39 (3) (2009) 533-540.

[4] F. Barcellini, F. Détienne and J-M. Burkhardt, Distributed design and distributed social awareness: exploring intersubjective dimensions of roles, COOP 2010, Cooperative systems design, Aix en Provence, France, 2010, May 19-21.

[5] F. Barcellini, F. Détienne, J-M. Burkhardt and W. Sack, A socio-cognitive analysis of online design discussions in an Open Source Software community, Interacting With Computers, 20 (1) (2008) 141-165.

[6] S.L. Bryant, A. Forte and A. Bruckman, Becoming Wikipedian: transformation of participation in a collaborative online encyclopedia. Proceedings of the 2005 international ACM SIGGROUP conference on Supporting group work, Sanibel Island, Florida, USA. 2005, pp 1-10.

[7] L.L. Bucciarelli, Engineering design process, In: Dubinskas, F. (Ed.), Making time: culture, time and organization in high technology, Temple University Press, Philadelphia, PA, 1998, pp. 92-122.

[8] J-M. Burkhardt, F. Détienne, A-M. Hébert and L. Perron, Assessing the "Quality of Collaboration" in TechnologyMediated Design Situations with Several Dimensions, INTERACT 2009, 12th IFIP TC13 Conference on HumanComputer Interaction, Uppsala, Sweden, 2009, August 24-28.

[9] J-M. Burkhardt and T. Lubart, T. Creativity in the age of emerging technology: Some issues and perspectives in 2010, CIM Journal 19 (2), (2010), 160-166.

[10] F. Détienne, Collaborative design : managing task interdependencies and multiple perspectives, Interacting With Computers, 18(1) (2006), 1-20.

[11] F. Détienne, M. Baker and J-M. Burkhardt, Analysing the quality of collaboration in task-oriented computer-mediated interactions: introduction to the workshop proceedings. Workshop « Analysing the quality of collaboration in taskoriented computer-mediated interactions », COOP 2010, Cooperative systems design, Aix en Provence, France, 2010, May 19-21. Online version « International Reports on SocioInformatics » (IRSI). http://www.iisi.de/102.0.html

[12] F. Détienne, J-F. Boujut and B. Hohmann, Characterization of Collaborative Design and Interaction Management Activities in a Distant Engineering Design Situation, In F. Darses, 
R.. Dieng, C. Simone, M. Zaklad (Eds) Cooperative Systems design, IOS Press, 2004, pp 83-98.

[13] F. Détienne, J-M. Burkhardt, A-M. Hébert and L. Perron, Assessing the quality of collaboration in design: bridging cognitive ergonomics and CSCL approaches, In Workshop " CSCW and Human Factors », CSCW'2008, 2008, November 9, San Diego, USA.

[14] D. Fréard, A. Denis, F. Détienne, M. Baker, M. Quignard and F. Barcellini, The role of argumentation in online epistemic communities: the anatomy of a conflict in Wikipedia, In Proc. of ECCE'10, Delft, the Netherlands, 2010.

[15] J.R. Hayes and L.S. Flower, Identifying the organization of writing processes, In L. W. Gregg \& E. R. Steinberg (Eds.), Cognitive processes in writing. Hillsdale, NJ: Lawrence Erlbaum, 2008.

[16] J.D. Herbsleb, Global Software Engineering: The Future of Socio-technical Coordination, In Future of Software Engineering (FOSE '07), IEEE Computer Society, Washington, DC, USA, 2007, pp 188-198.

[17] I. Jahnke, Dynamics of social roles in a knowledge management community, Computers in Human Behavior, 26(4) (2010), 533-546.

[18] G.M. Olson, J.S. Olson, M.R. Carter and M. Storrosten, Small Group Design Meetings: An Analysis of Collaboration, Human-Computer Interaction, 7 (1992), 347-374.

[19] P.B. Paulus. and B.A. Nijstad, Group creativity innovation through collaboration. Oxford University Press, 2003
[20] J. Roschelle and S.D. Teasley, The construction of shared knowledge in collaborative problem solving, In: O'Malley C. (Ed.) Computer Supported Collaborative Learning, Berlin: Springer-Verlag, 1995, pp. 69-97.

[21] W. Sack, F. Détienne, N. Ducheneaut, J-M. Burkhardt, D. Mahendran and F. Barcellini, A methodological framework for socio-cognitive analyses of collaborative design of Open Source Software, JCSCW, 15 (2-3) (2006), 229-250.

[22] S. Safin, A. Verschuere, J-M. Burkhardt, F. Détienne and AM. Hébert, Quality of collaboration in a distant collaborative architectural educational setting, Workshop « Analysing the quality of collaboration in task-oriented computer-mediated interactions ", COOP 2010, Cooperative systems design, Aix en Provence, France, 2010, May 19-21. Online version « International Reports on Socio-Informatics » (IRSI). http://www.iisi.de/102.0.html

[23] H. Spada, A. Meier, N. Rummel and S. Hauser, A new method to assess the quality of collaborative process in CSCL, In T. Koschmann, D. Suthers \& T. W. Chan (Eds.), Computer Supported Collaborative Learning 2005: The Next 10 Years! Mahwah, NJ: Lawrence Erlbaum Associates, 2005, pp. 622631.

[24] W. Visser, The cognitive artifacts of designing, Mahwah, NJ: Lawrence Erlbaum Associates, 2006.

[25] H.T. Welser, E. Gleave, D. Fisher and M. Smith, Visualizing the Signatures of Social Roles in Online Discussion Groups. Journal of Social Structure, 8 (2) (2007). 